\title{
An Approach to Boundary Extraction of Palm Lines and Vein Pattern
}

\author{
Mr. S. D. Raut \\ School of Computational Sciences, Solapur University, Solapur. Maharashtra, India. \\ profraut@ymail.com \\ Dr. V. T. Humbe \\ School of Technology, S.R.T.M. U. N. Sub-Campus, Latur, Maharashtra, India. \\ vikashumbe@gmail.com
}

\begin{abstract}
The palm vein biometrics is automated tool to recognize a person based on human vein pattern. The vein pattern is intrinsic and subcutaneous so that is very difficult to forge or fake. This paper discusses about the feature extraction of the hand based recognition system that involves features like vein pattern, principal lines and secondary lines. The morphological operations such as opening, closing and edge detection technique like canny algorithm are used to extract the feature set. The result shows the prominent feature extraction using image processing techniques.
\end{abstract}

Index Terms-Opening, Closing, Canny edge, Vein pattern, features extraction.

\section{INTRODUCTION}

Biometrics is a system tool to recognize a person based on physiological or behavioral trait. The physiological traits are the face [1], finger [2], hand [3], knuckle [4] and palm [5] etc. The behavioral traits are the voice [6], keystroke [7], handwritten character [8] and signature [9]. The research is trading towards the human vein characteristics. The vein is a blood vessel; which carries de-oxygenated blood and lies beneath the skin [10]. The pattern of this blood vessel is unique and distinct from person to person [11] as shown cross sectional view of the skin in fig. 1. Even the identical twins have the different, distinct and unique vein pattern [11]. The imaging techniques are evolved a lot to capture the blood vessel pattern lies under the skin. The vein pattern acquisition has done by illuminating the red, green, blue and near infrared light [12]. The de-oxygenated blood carried by the vein can be scanned using such illuminations [15].

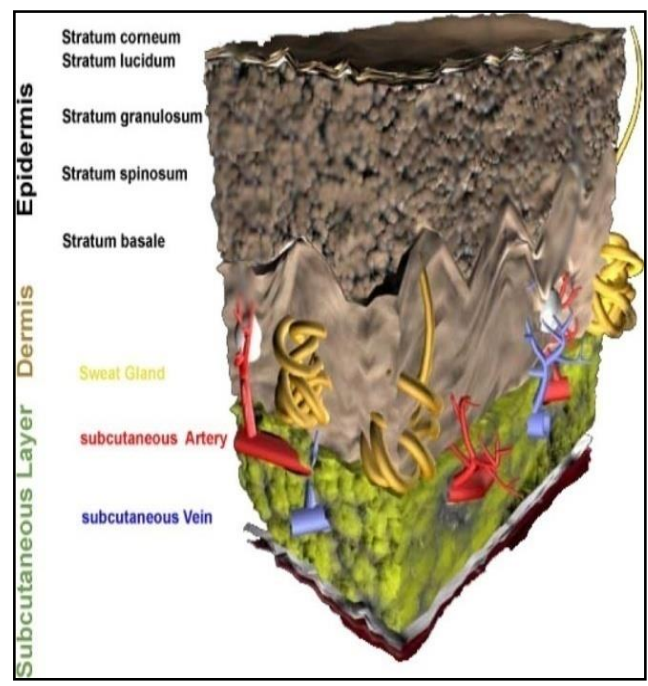

Fig 1. A model to show Cross-sectional view of the skin.

The researcher found that the near infrared light at $880-930 \mathrm{~nm}$ results to show the subcutaneous vein pattern [10] [15]. The paper discusses about the existing database developed by the Hong Kong Polytechnic University named as Multispectral Palm print Image database. The images of this database are experimented under proposed method includes two step phases I and II mainly focuses on image enhancement [13], morphological image processing and edge detection algorithm. The aim of the experiment is to extract the vein pattern and principal, secondary lines and edges from an image.

\section{IMAGE DATABASE}

For our study, we have obtained an access of existing 
image database. The database is developed by Biometric Research Centre of Polytechnic Hong Kong University. They have developed an image acquisition device as shown in fig 2; which can capture palm print image under red, green, blue and near infrared illumination as shown in fig 3. This database is named as multispectral Palm print image database [15].

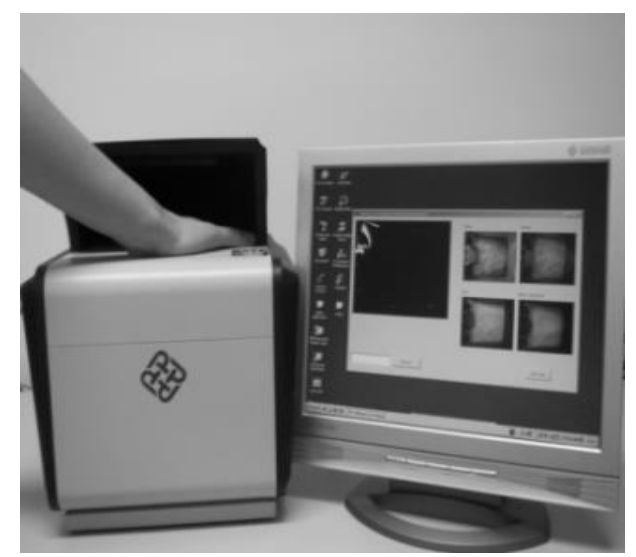

Fig 2. POLYU Image Acquisition Device

As a part of enrollment; about 250 individuals of ages between 20 to 60 years, have been registered. Out of 250 individuals, 195 males and 55 were females. They have conducted enrollment in two sessions of 9 days duration. They have applied a region of interest extraction algorithm and devised a palm print image as shown in fig 3.

Each part of their database was named as " $\mathrm{xxxx"}$ ". "xxxx" stand for the individuality of the person ( 1 to 500 sample). In their database, the first 6 images (1_xx) were acquired in the first session and then 6 images (2_xx) were acquired in the second session, "xx" indicates the image index for their given session (1 to 6 samples). Out of these multispectral palm print images, for our study we have performed experiments on palm print image captured under near infrared illumination fig. 3 (d).

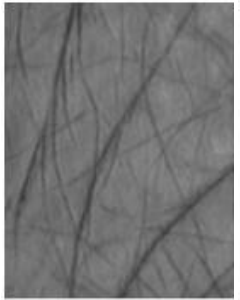

(a)

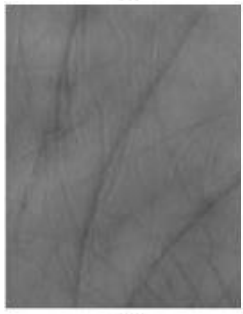

(c)

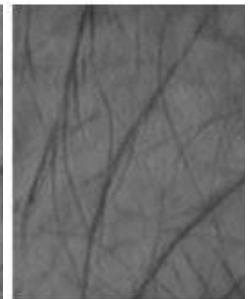

(b)

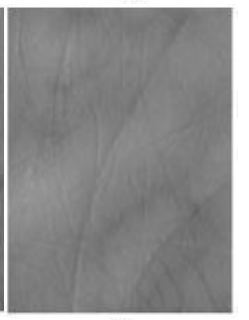

(d)
Fig.3. Palm prints image under light illumination as (a) red, (b) green, (c) blue and (d) near infrared.

\section{IMAGE ENHANCEMENT}

The principal objective of enhancement is to process an image so that the result is more suitable than the original image for a specific application. Visual evaluation of image quality is a highly subjective process. Spatial domain methods are procedures that operate directly on these pixels. Spatial domain processes [13] [14] will be denoted by the expression:

$$
g(x, y)=T[f(x, y)]
$$

Where $f(x, y)$ is the input image, $g(x, y)$ is the processed image, and ' $\mathrm{T}$ ' is an operator on, defined over some neighborhood of $(\mathrm{x}, \mathrm{y})$. The simplest form of ' $\mathrm{T}$ ' is when the neighborhood is of size $1 \times 1$ (that is, a single pixel).In this case, ' $g$ ' depends only on the value of at ( $x$, $\mathrm{y}$ ), and ' $\mathrm{T}$ ' becomes a gray-level (also called an intensity or mapping) transformation function of the form $\mathrm{S}=\mathrm{T}(\mathrm{r})$.

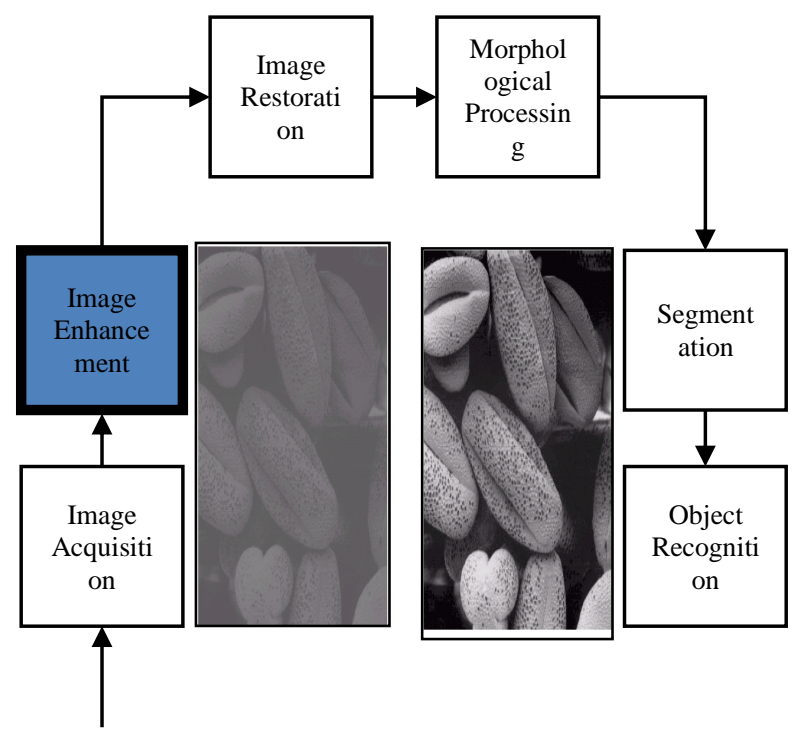

Problem Domain

Fig 4. Image Enhancement: key stage in Digital Image Processing.

\section{METHODOLOGY}

The morphological operations are the part of our study. Whereas, it is actually branch of image processing useful for analyzing shapes in images. The morphological operation open is said to happen; when erosion operation is followed by the dilation. It can be represented as follows [14]:

Suppose A and B are the set of pixels.

$$
\bigoplus A \quad B=\{(x, y)+(u, v):(x, y) \varepsilon A(u, v) \varepsilon B\}
$$

Given a set $A$ and $B$, the erosion of $A$ by $B$, defined as:

$$
A \theta B=\left\{w: B_{w} \sqsubseteq A\right\}
$$

This means erosion of $\mathrm{A}$ by $\mathrm{B}$ consists of all points $\mathrm{w}=$ $(\mathrm{x}, \mathrm{y})$ for which $\mathrm{Bw}$ is in $\mathrm{A}$. 


$$
A o B=(A \oplus B) B \text {. }
$$

That is $\mathrm{A}$ o B is the union of all translations of $\mathrm{B}$ which fit inside the A.

After these morphological operations; we had performed the edge detection of the image. The canny edge detection algorithm includes steps like Smoothing; Finding gradients; Non-maximum suppression; double thresholding and Edge tracking by hysteresis [14].

\section{EXPERIMENT AND RESULTS}

The experiments were carried on palm vein image captured under near infrared illumination. The operations such as image enhancement opening and closing followed by canny edge detection were performed so as to gain and extract the palm vein pattern lies at palm region of hand. The algorithm for the experimentation is as follows:

1. Input and Read the image from POLYU multispectral image database.

2. Apply image enhancement operation to adjust the image intensity values or color-map.

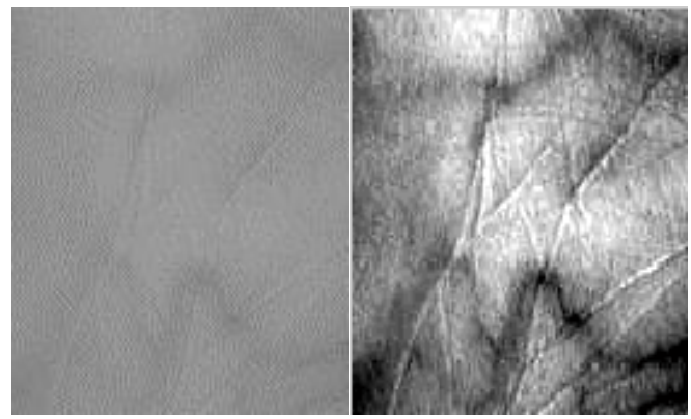

(a)

(b)

Fig 5. (a) Original image; (b) intensity values adjusted image

3. Apply image enhancement operation on output of step 2 to enhance the contrast using histogram equalization.

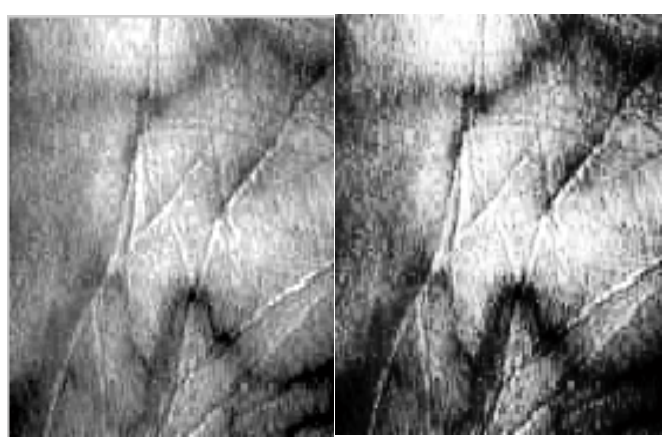

(a)

(b)

Fig. 6. (a) intensity values adjusted image; histogram equalized image of (a)

4. Convert the histogram equalized image into binary image. This will result into obtain the vein pattern and some of the principal line in a region.

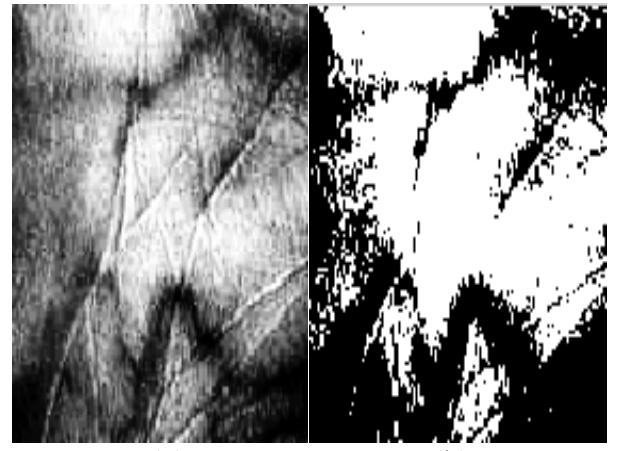

(a)

(b)

Fig 7. (a) Histogram equalized image; (b) Binary image of (a)

5. Perform closing morphological operation.

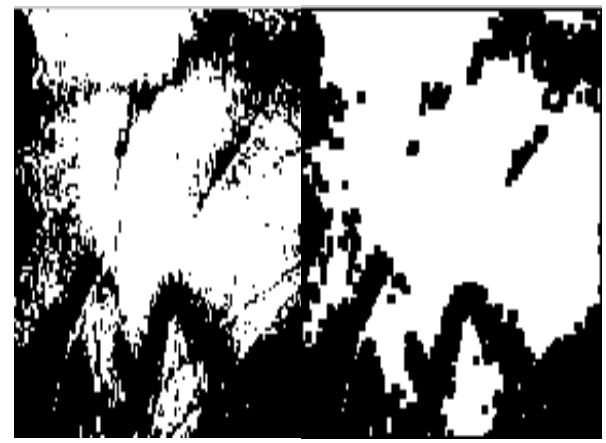

(a) (b)

Fig 8. (a) Binary image; (b) Morphed image through closing

6. Perform opening morphological operation on the output of step 5 .

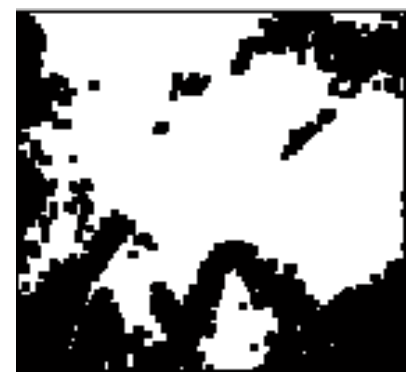

(a)

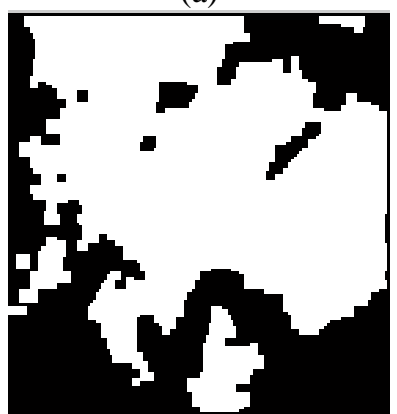

(b)

Fig 9. (a) Morphed image through closing; (b) Morphed image through opening on (a)

These are the first phase of our experiments. The sequence of experiments was carried so as to obtain the palm vein pattern. 
7. Apply canny edge detection algorithm on histogram equalized as output of step 3. As we adjust the threshold of edge detection, will result into an image which shows the edges of principal lines, secondary lines and blood vessel or vein.

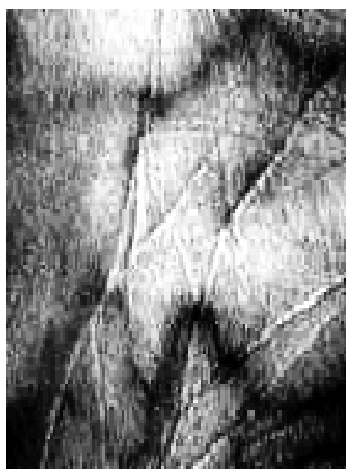

(a)

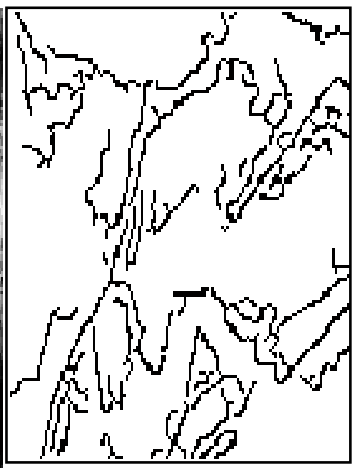

(b)
Fig 10. (a) Histogram equalized image; Canny edge detection of (a).

This is said to be second phase of experiment. Which leads us to gain the edge of the vein pattern lies at the palm region of hand.

8. Fusion of phase I and phase II of experiment lead us to show exactly the palm vein pattern, edges of it and principal lines too.

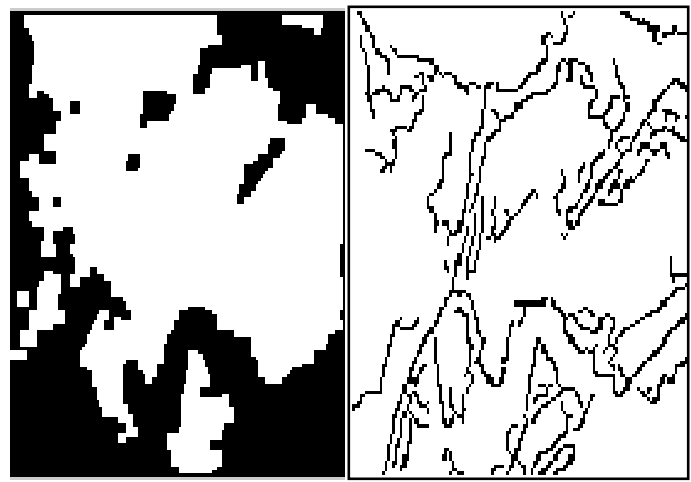

(a) (b)

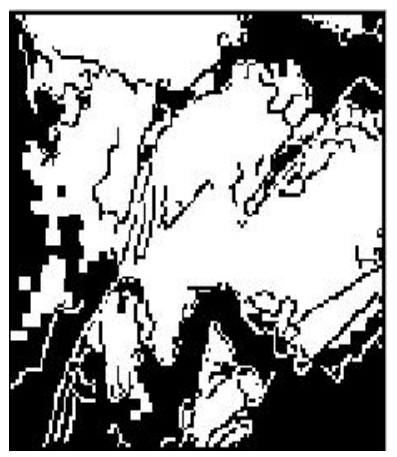

(c)

Fig 11. (a) Morphed image; (b) Canny edge image; Fusion of (a) and (b)

The result shows the experiment was successful. Through this experimentation we had obtained the edge palm vein pattern, principal line and secondary lines too as shown in fig. 10.
The obtained result can be interpreted as; when image sampled under image processing techniques in two different phases such as Phase-I includes enhancement, image domain conversion, morphological operations and Phase-II includes Edge detection. Then finally the fusion of the two phases to extract the vein pattern and principal lines from an image. The enhancement operations such as to adjust the color map and equalize the histogram will make the image visually appealing and also highlight the vein pattern while darkening the veins from the image. The enhanced image is inputted to image domain conversion operation that is to convert image enhanced image into binary form. Then this image is experimented under morphological operation that is closing (dilation followed by erosion) and output of this is experimented under opening that is (erosion followed dilation). Another phase of experimentation includes the output of enhancement operations to be given to Canny Edge detection algorithm. The experimentation is summarized in fig. 12 as an experimentation algorithm.

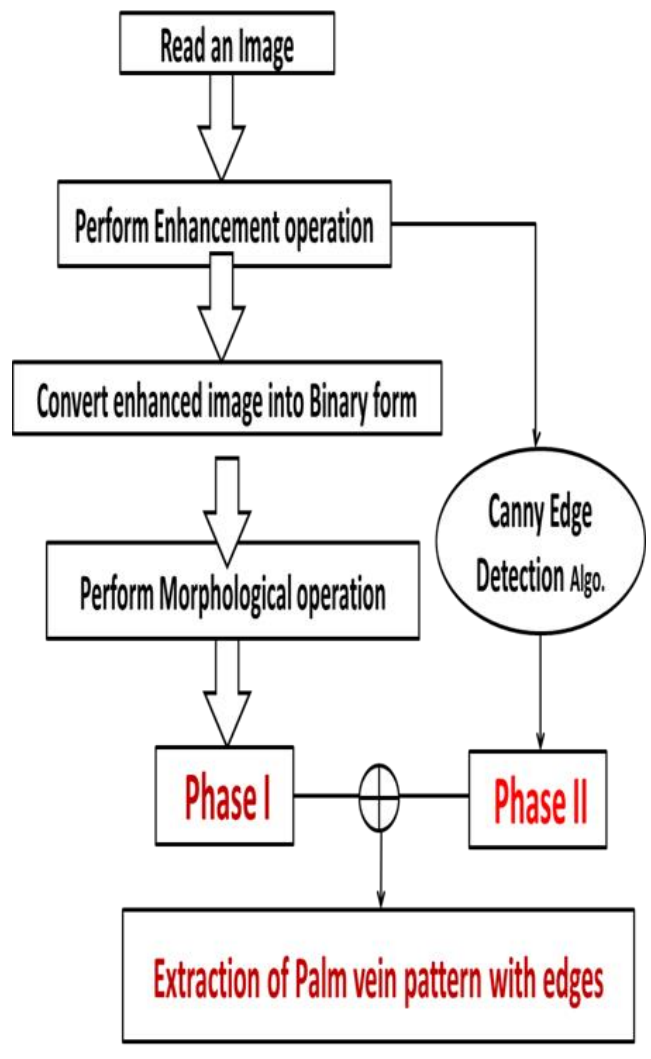

Fig 12. Flow Chart of Vein Pattern and Edge detection

\section{CONCLUSION}

The palm vein biometrics is today's attraction for the budding researcher. It is well known for the uniqueness and acceptability for recognition. The hemoglobin in the blood vessel is scanned using near infrared light illumination peaking at 880-930nm. For our study and implementation; we have obtained the multispectral palm print image database devised by the Hong Kong Polytechnic University (POLYU). The part of our methodology was based on image processing techniques 
such as image enhancement, morphological operations and edge detection technique. By using these tools; our objective was to obtain the palm feature such as vein pattern, principal and secondary lines and edges of these. As per the experiment and results we had done with the two phases of experiments; in order to extract the palm vein pattern and principal line based on morphological and edge detection operation.

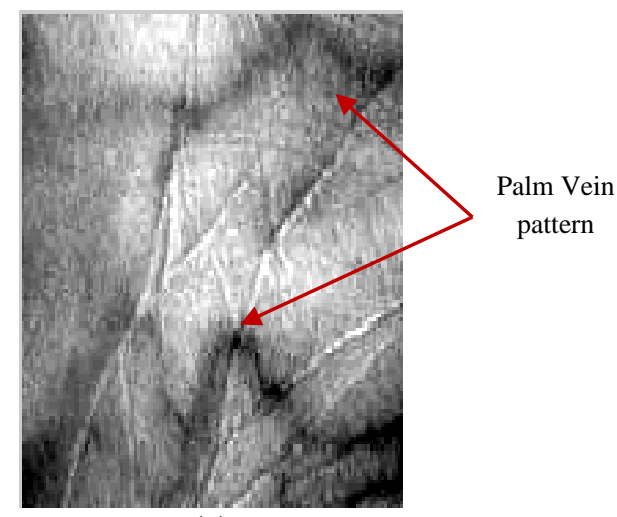

(a)

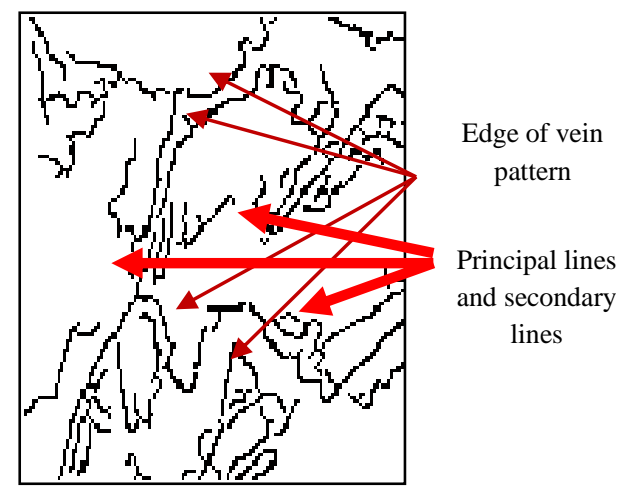

(b)

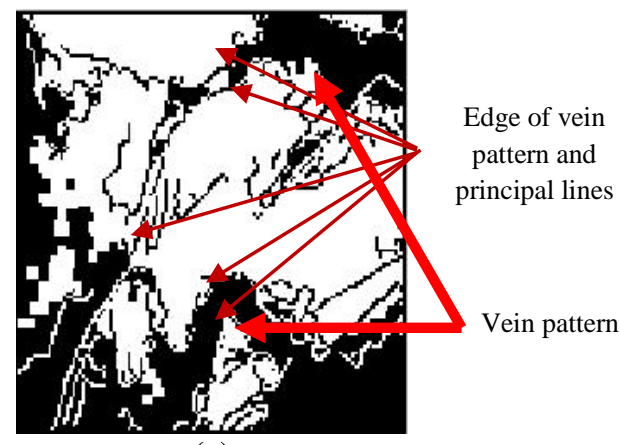

(c)

Fig 13. (a) - (c) Analysis of the images resulted through the experimentation.

\section{ACKNOWLEDGEMENT}

We are thankful to School of Computational Sciences of Solapur University, Solapur and School of Technology of S.R.T.M.U.N., Sub-campus Latur for providing us well equipped Computer Lab and infrastructure to do our experiments and study. We also thank to Biometric Research Centre of Polytechnic Hong Kong University for providing us an access of image database for our research work.

\section{REFERENCES}

[1] Sami Romdhani, Volker Blanz, and Thomas Vetter, "Face recognition based on fitting a 3D morphable model", European Conference on Computer Vision, Springer, 2002.

[2] Vikas Humbe, S. S. Gornale, Ramesh Manza, K.V. Kale, "Mathematical Morphology Approach for Genuine Fingerprint Feature Extraction", International Journal of Computer Science and Security, Vol 1, Issue 2, 2007.

[3] Feng-Sheng Chen, Chih-Ming Fu, Chung-Lin Huang, "Hand gesture recognition using a real-time tracking method and hidden Markov models", Image and Vision Computing, Pp. 745-758, Elsevier Science, 2003.

[4] Lin Zhang, Lei Zhang, and David Zhang, "FingerKnuckle-Print Verification Based on Band-Limited Phase-Only Correlation", CAIP 2009, LNCS 5702, pp. 141-148, Springer, 2009.

[5] Maurício Ramalho, Sanchit Singh, Paulo Lobato Correia and Luís Ducla Soares, "Secure Multi-Spectral Hand Recognition System", European Signal Processing Conference, Pp. 2269-2273.

[6] Richard P. Lippmann, "Speech recognition by machines and humans", Speech Communication Pp.1-15, Elsevier Science, 1997.

[7] Fabian Monrosea; Aviel D. Rubinb, "Keystroke dynamics as a biometric for authentication", Future Generation Computer Systems, Pp. 351-359, Elsevier Science, 2000.

[8] Rahul Kala, Harsh Vazirani, Anupam Shukla And Ritu Tiwari, "Offline Handwriting Recognition using Genetic Algorithm", International Journal of Computer Science Issues, Vol. 7, Issue 2, No 1, March 2010.

[9] O.C Abikoye, M.A Mabayoje, R. Ajibade, "Offline Signature Recognition \& Verification using Neural Network", International Journal of Computer Applications, Vol 35 No 02, Pp.44-51, 2011.

[10] S. D. Raut, V. T. Humbe, "Review of Biometrics: Palm Vein Recognition System", IBMRD's Journal of Management and Research, Vol. 03, Issue 01, E-ISSN: 2348-5922, Print ISSN: 2277-7830, March 2014.

[11] Kumar, A., K.V. Prathyusha, "Personal authentication using hand vein triangulation and knuckle shape" IEEE Transactions on Image Processing, vol. 38, issue 9, 2009.

[12] D. Zhang, Z. Guo, Guangming Lu, Lei Zhang, Wangmeng Zuo, "An Online System of Multispectral Palm print verification", IEEE Transaction on Instrumentation and Measurement, vol. 59, issue. 02, Pp. 480-490, 2010

[13] V. P. Zharov, S. Ferguson, J. F. Eidt, P. C. Howard, L. M. Fink, and M. Waner, "Infrared imaging of subcutaneous veins", Lasers Surg. Med., vol. 34, no. 1, pp. 56-61, Jan. 2004.

[14] Shriram D. Raut, Vikas T. Humbe, "Statistical Analysis of Resulting Palm vein Image through Enhancement Operations", International Journal of Information Engineering and Electronics Business, vol.5, no.6, Pp. 4754, ISSN: 2074-9023(Print), 2074-9031(Online), Dec, 2013.

[15] Rafael C. Gonzalez. "Digital Image Processing" Second Edition, University of Tennessee Richard E. Woods, MedData Interactive.

[16] David Zhang, Zhenhua Guo, Guangming Lu, Lei Zhang, Wangmeng Zuo, "An Online System of Multispectral Palm print verification", IEEE Transaction on Instrumentation and Measurement, vol. 59, issue. 02, pp. 480-490, 2010. 


\section{Author Profile}

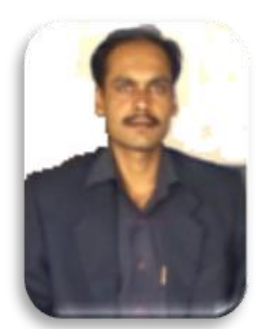

Dr. Vikas T. Humbe has completed his Ph.D. degree from University Department of Computer Science and Information Technology, Dr.B.A.M.U. Aurangabad and working as the Assistant Professor at Department of Computer Science, School of Technology, S.R.T.M. University, Nanded, Sub-campus, Latur. In teaching, he has been focusing on Digital Image and video processing concepts and Problem Based Learning approaches in Computer Science Education. In research, his current interests include Pattern Recognition, Image and video based processing, Data ware housing and web mining etc. He has published 42 research articles at National/International conferences and journals also he is the author of the books. He is reviewer for various International and National Journals and Conferences like Elsevier's Pattern Recognition Letters, Journal on Machine Vision and Applications, Academic Journals, IEEE IJCNN 07 and 09, ACVIT-09 etc. He is Member of IACSIT Singapore, Member of IAEng, Hong Kong, CSTA, USA and Graduate
Member of IEEE USA. His area of research interest is Biometrics, Image Processing, Computer Vision and Video Processing. He is the IEEE Graduate Member and has immense research recognition worldwide.

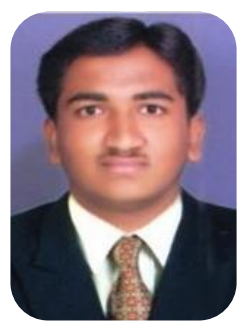

Mr. Shriram D. Raut is pursuing his Ph.D degree in Computer Science. He has completed his M.Sc. degree in Computer Science from University Department of Computer Science and Information Technology from Dr.B.A.M.U. Aurangabad and is working as the Assistant Professor at Department of Computer Science and Application, School of Computational Sciences, Solapur University, Solapur. He is Member of IACSIT Singapore, Member of IAEng, Hong Kong, and CSTA, New York. He worked as the Research Scholar and submitted a project in Computer Science under the UGC SAP (II) DRS Phase-I: 2009-2014 under the theme "Biometric: Multimodal System Development”.

How to cite this paper: S. D. Raut, V. T. Humbe,"An Approach to Boundary Extraction of Palm Lines and Vein Pattern", IJIGSP, vol.6, no.12, pp.47-52, 2014.DOI: 10.5815/ijigsp.2014.12.07 